Polymer Journal, Vol. 7, No. 6, pp 604-612 (1975)

\title{
Theory of Diffusion-Controlled Intrachain Reactions of Polymers
}

\author{
Seiichi Sunagawa and Masao DoI \\ Department of Physics, Faculty of Science, Tokyo Metropolitan University, \\ Setagaya-ku, Tokyo, Japan.
}

(Received March 25, 1975)

\begin{abstract}
The reaction rates for the diffusion-controlled ring closure reaction of polymers are calculated exactly based on the harmonic spring model. This is done in the limit of infinitely high intrinsic rate constant. The results are compared with those obtained by the closure approximation of Wilemski and Fixman. Both are found to be in relatively good agreement. The validity of their approximation is discussed. Finally, a simple physical interpretation is given for the present results.

KEY WORDS Intrachain Reaction / Diffusion-Controlled / Reaction Rate / Closure Approximation / Harmonic Spring Model / Ring Chain /
\end{abstract}

Recently, the use of chemical reactions to investigate the conformational and dynamical behavior of polymer chains has been suggested by a number of authors. Theoretical attempts to calculate the intrachain reaction rate have been made by Wang and Davidson ${ }^{1}$, Goodman and Morawetz ${ }^{2}$ and Sisido ${ }^{3}$ who discussed the reactions of polymers with small intrinsic rate constants. In this case, the conformational distribution of the polymer is considered to be almost in equilibrium, so that the calculation of reaction rate is reduced to the evaluation of the probability of finding a polymer chain in a certain conformation for the reaction to take place. On the other hand, if the intrachain reaction is intrinsically very fast, the rate limiting process will be the conformational change which brings potentially reactive groups into contact, and the reaction then becomes diffusion-controlled.

A general theory of diffusion-controlled intrachain reactions of polymers has been recently developed by Wilemski and Fixman. ${ }^{4}$ To obtain a tractable expression for the reaction rate they assumed that the conformation of a polymer chain in a reaction region is in equilibrium. They then obtained a closed equation for the reaction rate and performed extensive numerical calculations for a several polymer models. Their closure approximation seems to be plausible in case of small intrinsic rate constants, but this is not so apparent in the opposite case because the distribution of unreacted polymers is distorted from the equilibrium by the occurrence of the reaction.

In the present paper, we attempt to examine the validity of their closure approximation. In order to do this, we discuss the reaction rate in the limit of infinitely high intrinsic rate constant using a simple model. In our reaction system, a polymer chain is represented by a harmonic spring model to the ends of which a pair of reactive groups are attached and reaction occurs whenever the end segments touch the surface of an effective reaction sphere of radius $R$. The conventional method to calculate the reaction rate for this system is to solve the diffusion equation under the Smoluchowski boundary condition. ${ }^{5}$ However, this is not easy for the case of a harmonic spring model and hence, in this paper, we employ a new approach.

We first introduce a sink term into the diffusion equation and rewrite it in the form of an integral equation. Owing to the spherical symmetry of the system, the integral equation is rigorously solved. The reaction rates are then calculated as a function of $\gamma=\sqrt{3 / 2}(R / L)$, where $L^{2}$ is a mean square length of the spring. This is done numerically for several values of $\gamma$. Calculation is also performed analytically in the limit of $\gamma \rightarrow 0$.

The results are then compared with those obtained by the closure approximation of Wilemski and Fixman and the validity of their approximation is discussed. We also give a simple 
physical interpretation for the present results in connection with the theory of a free particle system.

\section{FORMAL EXPRESSION FOR THE REACTED FRACTION}

Since we are considering the intramolecular reaction of an isolated chain, the system is described only by the relative position vector $\boldsymbol{r}$ between the two reactive segments. The diffusion equation for the distribution function $P(r, t)$ of the system is then written as

$$
\begin{gathered}
\frac{\partial}{\partial t} P(\boldsymbol{r}, t)=\mathscr{L}(\boldsymbol{r}) P(\boldsymbol{r}, t) \\
\mathscr{L}(\boldsymbol{r})=D \frac{\partial}{\partial \boldsymbol{r}}\left(\frac{1}{k T} \frac{\partial U}{\partial \boldsymbol{r}}+\frac{\partial}{\partial \boldsymbol{r}}\right)
\end{gathered}
$$

In eq $2, D$ is the relative diffussion constant of the segments and $U$ is the potential energy of the spring given by

$$
U(\boldsymbol{r})=\frac{3 k T}{2 L^{2}}|\boldsymbol{r}|^{2}
$$

Our problem is to solve eq 1 under the boundary condition

$$
P(r, t)=0 \quad \text { at } \quad|r|=R
$$

Instead of considering this boundary condition, we introduce a sink term $\xi(R, t)$ defined in relation to the surface of the reaction sphere into the diffusion eq 1

$$
\frac{\partial}{\partial t} P(\boldsymbol{r}, t)=\mathscr{L} P-\int_{\partial v} \mathrm{~d}^{2} R \delta(\boldsymbol{r}-\boldsymbol{R}) \xi(\boldsymbol{R}, t)
$$

where subscript $\partial v$ denotes an integration over the surface of the reaction sphere. The function $\xi(\boldsymbol{R}, t)$ represents the reaction rate on the surface of the reaction sphere per unit area.

In terms of the Green function for the diffusion operator $\mathscr{L}$, a formal solution of eq 5 can be written as

$$
\begin{aligned}
P(\boldsymbol{r}, t)= & \int_{v} \mathrm{~d}^{3} r^{\prime} G\left(\boldsymbol{r}, \boldsymbol{r}^{\prime}, t\right) P\left(\boldsymbol{r}^{\prime}, 0\right) \\
& -\int_{0}^{t} \mathrm{~d} s \int_{\partial v} \mathrm{~d}^{2} R^{\prime} \int_{v} \mathrm{~d}^{3} r^{\prime} G\left(\boldsymbol{r}, \boldsymbol{r}^{\prime}, t-s\right) \\
& \times \delta\left(\boldsymbol{r}^{\prime}-\boldsymbol{R}^{\prime}\right) \xi\left(\boldsymbol{R}^{\prime}, s\right)
\end{aligned}
$$

where subscript $v$ denotes an integration over the outer space of the reaction sphere and the Green function $G$ is given by ${ }^{4}$

$$
\begin{aligned}
\boldsymbol{G}\left(\boldsymbol{r}, \boldsymbol{r}^{\prime}, t\right)= & \left\{\frac{2 \pi L^{2}}{3}\left(1-\mathrm{e}^{-6 D t / L^{2}}\right)\right\}^{-3 / 2} \\
& \times \exp \left\{-\frac{3}{2 L^{2}} \frac{\left|\boldsymbol{r}-\boldsymbol{r}^{\prime} \mathrm{e}^{-3 D t / L^{2}}\right|^{2}}{\left(1-\mathrm{e}^{-6 D t / L^{2}}\right)}\right\}
\end{aligned}
$$

We assume that the system is in equilibrium at time $t=0$.

$$
P(\boldsymbol{r}, 0)=P_{\mathrm{eq}}(\boldsymbol{r})=\left(\frac{2 \pi L^{2}}{3}\right)^{-3 / 2} \exp \left(-\frac{3}{2 L^{2}}|\boldsymbol{r}|^{2}\right)
$$

Function $\xi(\boldsymbol{R}, t)$ is determined so that $P(\boldsymbol{r}, t)$ in eq 5 satisfies the boundary condition (4)

$$
\begin{aligned}
& \int_{v} \mathrm{~d}^{3} r^{\prime} G\left(\boldsymbol{R}, \boldsymbol{r}^{\prime}, t\right) P\left(\boldsymbol{r}^{\prime}, 0\right) \\
= & \int_{0}^{t} \mathrm{ds} \int_{\partial v} \mathrm{~d}^{2} R^{\prime} \int_{v} \mathrm{~d}^{3} r^{\prime} G\left(\boldsymbol{R}, \boldsymbol{r}^{\prime}, t-s\right) \\
& \times \delta\left(\boldsymbol{r}^{\prime}-\boldsymbol{R}^{\prime}\right) \xi\left(\boldsymbol{R}^{\prime}, s\right) \\
= & \int_{0}^{t} \mathrm{~d} s \int_{\partial v} \mathrm{~d}^{2} R^{\prime} G\left(\boldsymbol{R}, \boldsymbol{R}^{\prime}, t-s\right) \xi\left(\boldsymbol{R}^{\prime}, s\right)
\end{aligned}
$$

Thus the boundary value problem is reduced to an integral equation. This equation is in general difficult to solve. However, in the present case, a major simplification is possible. Since the system is spherically symmetrical, $\xi(\boldsymbol{R}, s)$ does not depend on $\boldsymbol{R}$. Then, averaging eq 9 over the surface of the reaction sphere, we have

$$
A(t)=\int_{0}^{t} \mathrm{~d} s B(t-s) \xi(s)
$$

where

$$
\begin{gathered}
A(t)=\int_{\partial v} \mathrm{~d}^{2} R \int_{v} \mathrm{~d}^{3} r^{\prime} G\left(\boldsymbol{R}, \boldsymbol{r}^{\prime}, t\right) P_{\mathrm{eq}}\left(\boldsymbol{r}^{\prime}\right) \\
B(t)=\int_{\partial v} \mathrm{~d}^{2} R \int_{\partial v} \mathrm{~d}^{2} R^{\prime} G\left(\boldsymbol{R}, \boldsymbol{R}^{\prime}, \boldsymbol{t}\right)
\end{gathered}
$$

Since the integral eq 10 has the convolution form, its solution is immediately obtained by use of the Laplace transform. If we denote the Laplace transform of the function $g(t)$ by $\hat{g}(p)$, i.e.,

$$
\hat{g}(p)=\int_{0}^{\infty} \mathrm{d} t g(t) \exp (-p t)
$$

then

$$
\hat{\xi}(p)=\frac{\hat{A}(p)}{\hat{B}(p)}
$$

Thus $\xi(t)$ can be obtained if the inversion of eq 14 is performed. However, the procedure 
is very difficult to carry out analytically, so we have recourse to a numerical calculation. In that case, it is convenient to start from the original integral eq 10.

\section{NUMERICAL SOLUTION}

Throughout the calculation, the following dimensionless variables are adopted;

$$
\begin{gathered}
\gamma=\sqrt{\frac{3}{2}} \frac{R}{L} \\
\tau=\frac{6 D}{L^{2}} t
\end{gathered}
$$

In terms of the above-scaled variables, the Green function (7) is written as

$$
\begin{aligned}
\boldsymbol{G}\left(\boldsymbol{r}, \boldsymbol{r}^{\prime}, \tau\right)= & \left\{\frac{2 \pi L^{2}}{3}\left(1-\mathrm{e}^{-\tau}\right)\right\}^{-3 / 2} \\
& \times \exp \left\{-\frac{3}{2 L^{2}} \frac{\left|\boldsymbol{r}-\boldsymbol{r}^{\prime} \mathrm{e}^{-\tau / 2}\right|^{2}}{\left(1-\mathrm{e}^{-\tau}\right)}\right\}
\end{aligned}
$$

Hence, the integrals of eq 11 and 12 yield

$$
\begin{aligned}
A(\tau)= & \frac{4 \gamma^{3}}{\sqrt{\pi} R} \mathrm{e}^{-\gamma^{2}}-\frac{4 \sqrt{2} \gamma^{4}}{\pi R}\left(\operatorname{cosech} \frac{\tau}{2}\right)^{1 / 2} \mathrm{e}^{3 \tau / 4} \\
& \times \exp \left(-\frac{\gamma^{2}}{2} \mathrm{e}^{\tau / 2} \operatorname{cosech} \frac{\tau}{2}\right) F(\alpha, \beta) \\
B(\tau)= & 4 \sqrt{2 \pi} R \gamma \mathrm{e}^{3 \tau / 4}\left(\operatorname{cosech} \frac{\tau}{2}\right)^{1 / 2} \\
& \times \exp \left(-\gamma^{2} \operatorname{coth} \frac{\tau}{2}\right) \sinh \left(\gamma^{2} \operatorname{cosech} \frac{\tau}{2}\right)
\end{aligned}
$$

where

$$
\begin{gathered}
F(\alpha, \beta)=\int_{0}^{1} \mathrm{~d} x x \mathrm{e}^{-\alpha x^{2}} \sinh \beta x \\
\alpha=\frac{\gamma^{2}}{2} \mathrm{e}^{\tau / 2} \operatorname{cosech} \frac{\tau}{2} \\
\beta=\gamma^{2} \operatorname{cosech} \frac{\tau}{2}
\end{gathered}
$$

First, we shall investigate the short-time behavior of $A(\tau), B(\tau)$ and $\xi(\tau)$ and the long-time behavior of $A(\tau)$ and $B(\tau)$. These will be needed in the course of analysis.

If $\tau \ll 1$, eq 17 is simplified as

$$
G\left(\boldsymbol{r}, \boldsymbol{r}^{\prime}, \tau\right)=\left(\frac{3}{2 \pi L^{2} \tau}\right)^{3 / 2} \exp \left(-\frac{3\left|\boldsymbol{r}-\boldsymbol{r}^{\prime}\right|^{2}}{2 L^{2} \tau}\right)
$$

then, eq 11 and 12 yield

$$
\begin{gathered}
A(\tau)=\frac{1}{R}\left(\frac{2 \gamma^{3}}{\sqrt{\pi}}+\frac{2 \gamma^{2}}{\pi} \sqrt{\tau}\right) \\
B(\tau)=4 \sqrt{\pi} R \gamma \frac{1}{\sqrt{\tau}}
\end{gathered}
$$

Taking the Laplace transforms of eq 24 and 25 , we obtain

$$
\begin{gathered}
\hat{A}(p)=\frac{1}{R}\left(\frac{2 \gamma^{3}}{\sqrt{\pi}} \frac{1}{p}+\frac{\gamma^{2}}{\sqrt{\pi}} \frac{1}{p \sqrt{p}}\right) \\
\hat{B}(p)=4 \pi R \gamma \frac{1}{\sqrt{p}}
\end{gathered}
$$

then

$$
\hat{\xi}(p)=\frac{1}{R^{2}}\left(\frac{\gamma^{2}}{2 \pi \sqrt{\pi} \sqrt{p}}+\frac{\gamma}{4 \pi \sqrt{\pi} p}\right)
$$

Inversion of eq 28 gives

$$
\begin{aligned}
\xi(\tau) & =\frac{1}{2 \pi i} \int_{-i \infty}^{i \infty} \mathrm{d} p \hat{\xi}(p) \mathrm{e}^{p \tau} \\
& =\frac{1}{R^{2}}\left(\frac{\gamma^{2}}{2 \pi^{2} \sqrt{\tau}}+\frac{\gamma}{4 \pi \sqrt{\pi}}\right)
\end{aligned}
$$

Note that $\xi(\tau)$ has the same time-dependence as that of a free particle system. This is natural because eq 23 has the same form as that of the free particle.

To investigate the long-time behavior of $A(\tau)$ and $B(\tau)$, we make use of the eigenfunction expansion of the Green function;

$$
\boldsymbol{G}\left(\boldsymbol{r}, \boldsymbol{r}^{\prime}, \tau\right)=P_{\mathrm{eq}}(\boldsymbol{r}) \sum_{n} \psi_{n}(\boldsymbol{r}) \psi_{n}\left(\boldsymbol{r}^{\prime}\right) \exp \left(-\lambda_{n} \tau\right)
$$

Here, $\lambda_{n}$ and $\psi_{n}$ are respectively eigenvalues and eigenfunctions of the operator $\mathscr{L}$;

$$
\begin{gathered}
\lambda_{n}=n \quad(n=0,1,2, \ldots) \\
\psi_{n}(\boldsymbol{r})=\sqrt{\frac{\sqrt{\pi} n !}{2 \Gamma(3 / 2+n)}} L_{n}{ }^{1 / 2}\left(3 \boldsymbol{r}^{2} / 2 L^{2}\right)
\end{gathered}
$$

where

$$
L_{n}^{1 / 2}(x)=\sum_{r=0}^{n}(-1)^{r}\left(\begin{array}{c}
n+1 / 2 \\
n-r
\end{array}\right) \frac{x^{r}}{r !}
$$

(the Laguerre polynomials)

In eq 30 , we have suppressed the angularly dependent terms because such terms do not contribute to the integrals of eq 11 and 12. The derivations of eq 31 and 32 are given in the Appendix.

By use of eq 30 , eq 11 and 12 are written as Polymer J., Vol. 7, No. 6, 1975 


$$
\begin{aligned}
& A(\tau)=\sum_{n=0}^{\infty} A_{n} \exp (-n \tau) \\
& B(\tau)=\sum_{n=0}^{\infty} B_{n} \exp (-n \tau)
\end{aligned}
$$

where

$$
\begin{aligned}
& A_{n}=\int_{\partial v} \mathrm{~d}^{2} R P_{\mathrm{eq}}(\boldsymbol{R}) \phi_{n}(\boldsymbol{R}) \int_{v} \mathrm{~d}^{3} r^{\prime} P_{\mathrm{eq}}\left(\boldsymbol{r}^{\prime}\right) \psi_{n}\left(\boldsymbol{r}^{\prime}\right) \\
& \boldsymbol{B}_{n}=\int_{\partial v} \mathrm{~d}^{2} R P_{\mathrm{eq}}(\boldsymbol{R}) \phi_{n}(\boldsymbol{R}) \int_{\partial v} \mathrm{~d}^{2} \boldsymbol{R}^{\prime} \psi_{n}\left(\boldsymbol{R}^{\prime}\right)
\end{aligned}
$$

The explicit forms of the first two terms are

$$
\begin{gathered}
A_{0}=\frac{4 \gamma^{3}}{\sqrt{\pi} R} \mathrm{e}^{-\gamma^{2}}-\frac{16 \gamma^{3}}{\pi R} \mathrm{e}^{-\gamma^{2}} F_{1}(\gamma) \\
A_{1}=\frac{24 \gamma^{3}}{\pi R} \mathrm{e}^{-\gamma^{2}}\left(\frac{3}{2} \gamma^{2}-1\right)\left\{F_{1}(\gamma)-\frac{2}{3} F_{2}(\gamma)\right\} \\
B_{0}=16 \sqrt{\pi} R \gamma^{3} \mathrm{e}^{-\gamma^{2}} \\
B_{1}=24 \sqrt{\pi} R \gamma^{3} \mathrm{e}^{-\gamma^{2}}\left(\frac{2}{3} \gamma^{2}-1\right)^{2} \\
F_{1}(x)=\int_{0}^{x} \mathrm{~d} y y^{2} \mathrm{e}^{-y^{2}}, \quad F_{2}(x)=\int_{0}^{x} \mathrm{~d} y y^{4} \mathrm{e}^{-y^{2}}
\end{gathered}
$$

Numerical results for $A(\tau)$ and $B(\tau)$ are shown in Figure 1 and Figure 2, respectively.

Our numerical procedure to solve eq 10 is as follows. As can be seen from eq 25 and 29, $B(\tau)$ and $\xi(\tau)$ are singular at $\tau=0$, so the analytic expressions, eq 25 and 29 , can be used in the time region from $\tau=0$ to some $\tau=\tau_{\mathrm{c}}$ where the short-time approximation holds. For the nonsingular parts, all functions are approximated by straight lines for each time-interval $\Delta$. Then eq 10 becomes

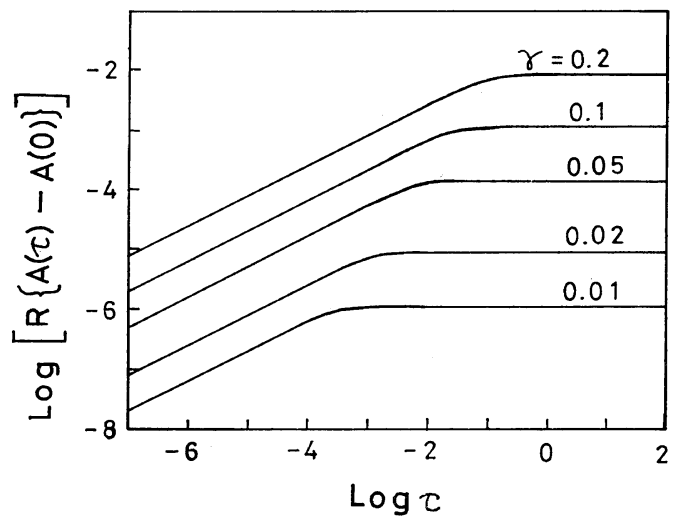

Figure 1. Time dependence of $A(\tau)$.

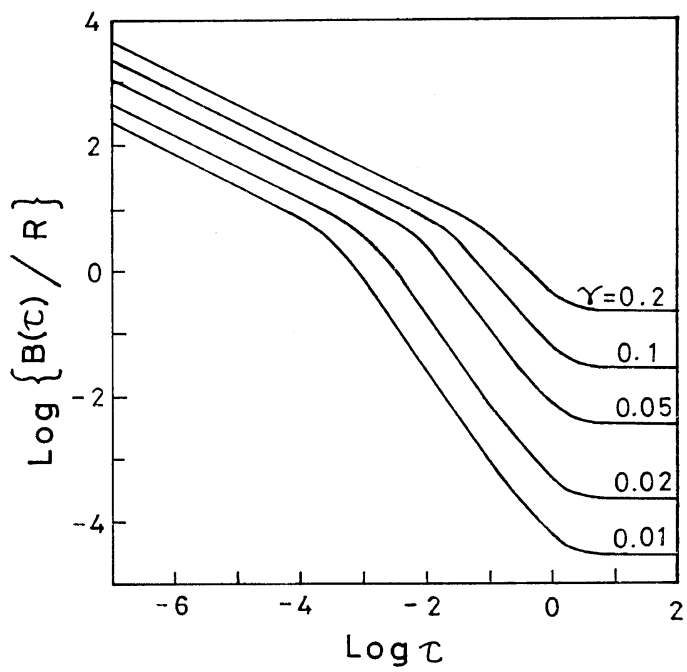

Figure 2. Time dependence of $B(\tau)$.

$$
\begin{aligned}
A(\tau)=\int_{0}^{\tau_{\mathrm{c}}} \mathrm{d} s B(\tau-s) \xi(s) & +\int_{\tau_{\mathrm{c}}}^{\tau-\tau_{\mathrm{c}}} \mathrm{d} s B(\tau-s) \xi(s) \\
& +\int_{0}^{\tau_{\mathrm{c}}} \mathrm{d} s B(s) \xi(\tau-s) \\
\equiv N_{1}+N_{2}+N_{3} &
\end{aligned}
$$

where

$$
\begin{aligned}
& N_{1}=\int_{0}^{\tau_{\mathrm{c}}} \mathrm{d} s\left[\frac{s}{\tau_{\mathrm{c}}}\left\{B\left(\tau-\tau_{\mathrm{c}}\right)-B(\tau)\right\}+B(\tau)\right] \\
& \times\left[\frac{1}{R^{2}}\left(\frac{\gamma^{2}}{2 \pi^{2} \sqrt{s}}+\frac{\gamma}{4 \pi \sqrt{\pi}}\right)\right] \\
& N_{2}=\sum_{l=0}^{m} \int_{0}^{\Delta} \mathrm{d} s\left[\frac{s}{\Delta}\left\{B\left(\tau-\tau_{l}-\Delta\right)-B\left(\tau-\tau_{l}\right)\right\}\right. \\
& \left.+B\left(\tau-\tau_{l}\right)\right]\left[\frac{s}{\Delta}\left\{\xi\left(\tau_{l}+\Delta\right)-\xi\left(\tau_{l}\right)\right\}+\xi\left(\tau_{l}\right)\right] \\
& N_{3}=\int_{0}^{\tau_{\mathrm{c}}} \mathrm{d} s \frac{4 \sqrt{\pi} \gamma R}{\sqrt{ } s}\left[\frac{s}{\tau_{\mathrm{c}}}\left\{\xi\left(\tau-\tau_{\mathrm{c}}\right)-\xi(\tau)\right\}+\xi(\tau)\right] \\
& \left(\tau_{0}=\tau_{\mathrm{c}}, \tau_{1}=\tau_{\mathrm{c}}+\Delta, \ldots, \tau_{\mathrm{m}}=\tau-\tau_{\mathrm{c}}-\Delta\right)
\end{aligned}
$$

If we set $\tau=\tau_{\mathrm{c}}+\Delta$ in eq $43, \xi\left(\tau_{\mathrm{c}}+\Delta\right)$ can be obtained in terms of the known functions $\mathrm{A}(\tau)$ and $B(\tau)$. Next, if we set $\tau=\tau_{\mathrm{c}}+2 \Delta, \xi\left(\tau_{\mathrm{c}}+2 \Delta\right)$ can be obtained from eq 43. Thus, for each time $\tau$, $\xi(\tau)$ can be evaluated by the repetition of the same procedure. As $\tau$ increases, $B(\tau)$ and $\xi(\tau)$ become more smooth, so we can choose larger time-interval $\Delta$. In this manner, $\xi(\tau)$ were calculated for various values of $\gamma$. The results are shown in Figure 3. 


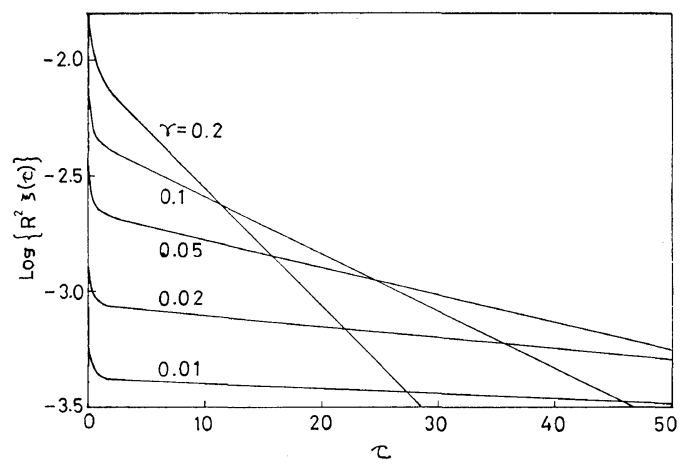

Figure 3. Time dependence of the reaction rate, $\xi(\tau)$.

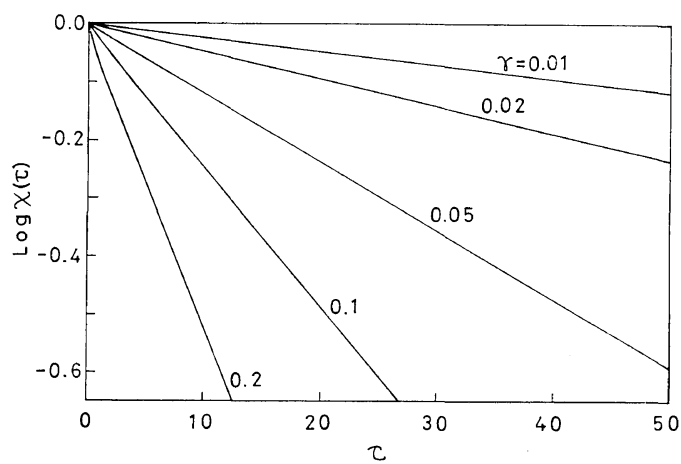

Figure 4. Time dependence of the unreacted fraction, $\chi(\tau)$.

The unreacted fraction of polymers, $\chi(\tau)$, is obtained from $\xi(\tau)$ as

$$
\chi(\tau)=1-4 \pi R^{2} \int_{0}^{\tau} \mathrm{d} s \xi(s)
$$

because $\xi(\tau)$ is the reaction rate per unit area of the reaction sphere. The numerical results for $\chi(\tau)$ are shown in Figure 4.

From Figures 3 and 4, we find that both $\xi(\tau)$ and $\chi(\tau)$ decay in a simple exponential law for almost the whole time region. As is understood from eq 47 , the decay rates of $\xi(\tau)$ and $\chi(\tau)$ are equal. The observed first-order reaction rate constant $k_{1}$ is given by this decay rate;

$$
\left.\begin{array}{l}
\xi(\tau)=\xi_{1} \exp \left(-k_{1} \tau\right) \\
\chi(\tau)=\chi_{1} \exp \left(-k_{1} \tau\right)
\end{array}\right\} \quad(\tau \gtrsim 1)
$$

Hence, $k_{1}$ can be evaluated from the slope of Figure 3 or 4 and is found to be very small compared to unity. However, this method is not appropriate for the purpose of the accurate evaluation of $k_{1}$. For that purpose, the following procedure is more convenient.

\section{THE REACTION RATE}

If we take the Laplace transforms of eq 34 and 35 and substitute them into eq 14 , we obtain

$$
\hat{\xi}(p)=\frac{\sum_{n=0}^{\infty} \frac{A_{n}}{p+n}}{\sum_{n=0}^{\infty} \frac{B_{n}}{p+n}}
$$

Then, the poles of $\hat{\xi}(p)$ are determined by the solutions of the equation

$$
\hat{B}(p)=\sum_{n=0}^{\infty} \frac{B_{n}}{p+n}=0
$$

because $p=-n$ gives no singularity of $\hat{\xi}(p)$. The roots of eq 50 are real and negative. This can be easily proved from the fact that all $B_{n}$ are real and positive. The observed reaction rate constant $k_{1}$ is given by the largest (or the smallest in the absolute value) root of eq 50 .

From the numerical results of the preceding section, the absolute value of the largest root of eq 50 is supposed to be very small. Hence, we expand eq 50 in power series of $p$

$$
\begin{aligned}
\hat{\boldsymbol{B}}(p)= & \frac{B_{0}}{p}+\sum_{n=1}^{\infty} \frac{B_{n}}{n}\left\{1-\frac{p}{n}+\left(\frac{p}{n}\right)^{2}\right. \\
& \left.-\left(\frac{p}{n}\right)^{3}+\left(\frac{p}{n}\right)^{4}-\cdots\right\} \\
= & \frac{C_{0}}{p}+C_{1}-C_{2} p+C_{3} p^{2}-C_{4} p^{3}+C_{5} p^{4}-\cdots=0
\end{aligned}
$$

where

$$
C_{0}=B_{0}, \quad C_{k}=\sum_{n=1}^{\infty} \frac{B_{n}}{n^{k}} \quad(k=1,2,3, \ldots)
$$

We solved eq 51 numerically by retaining the first six terms. In this case, we found that the convergence of the sum for $C_{1}$ is rather slow, so we evaluated $C_{1}$ by using the relation

$$
C_{1}=\sum_{n=1}^{\infty} \frac{B_{n}}{n}=\int_{0}^{\infty} \mathrm{d} \tau\left\{B(\tau)-B_{0}\right\}
$$

which is easily verified from eq 50 .

The results for $k_{1}$ are presented in Table I. 
Table I. The rate of reaction

\begin{tabular}{cc}
\hline$r$ & The rate of reaction $k_{1}$ \\
\hline 0.2 & 0.121 \\
0.1 & 0.0584 \\
0.05 & 0.0287 \\
0.02 & 0.0114 \\
0.01 & 0.00566
\end{tabular}

Note that $k_{1}$ increases almost in proportion to $\gamma$. From this observation, we found that the asymptotic form of $k_{1}$ in the limit of $\gamma \rightarrow 0$ can be calculated analytically. This will be discussed in the following section.

\section{ANALYTIC EXPRESSION FOR $\xi(\tau)$}

In the limit of small $\gamma$, we safely retain only the first two terms of eq 51 .

$$
\hat{B}(p)=\frac{C_{0}}{p}+C_{1}=0 \quad(\gamma \rightarrow 0)
$$

With eq 52 and 53 , eq 54 gives the largest pole of $\hat{\xi}(p)$ as

$$
k_{1}=\frac{B_{0}}{I}
$$

where

$$
I=\int_{0}^{\infty} \mathrm{d} \tau\left\{B(\tau)-B_{0}\right\}
$$

and $B_{0}$ is given by eq 40 .

Let us investigate the asymptotic form of $I$. By use of eq 19 and 40, I becomes

where

$$
I=4 \sqrt{\pi} R \gamma \mathrm{e}^{-\gamma^{2}} \int_{0}^{\infty} \mathrm{d} \tau F(\tau)
$$

$$
\begin{aligned}
F(\tau) & =\frac{\mathrm{e}^{\tau}}{\sqrt{\mathrm{e}^{\tau}-1}} \exp \left(-\frac{2 \gamma^{2}}{\mathrm{e}^{\tau}-1}\right) \\
& \times\left\{\exp \left(\frac{2 \gamma^{2} \mathrm{e}^{\tau / 2}}{\mathrm{e}^{\tau}-1}\right)-\exp \left(-\frac{2 \gamma^{2} \mathrm{e}^{\tau / 2}}{\mathrm{e}^{\tau}-1}\right)\right\}-4 \gamma^{2}
\end{aligned}
$$

At first sight, the asymptotic form of $I$ as $\gamma \rightarrow 0$ may seem to be obtained by expanding $F(\tau)$ in power series of $\gamma$. However, the coefficient of the first term in the expansion diverges. To overcome this difficulty, we introduce a new variable $y$ defined by

$$
\frac{2 \gamma^{2}}{3\left(\mathrm{e}^{\tau}-1\right)}=y
$$

and rewrite eq 57 as

$$
I=4 \sqrt{\pi} R \gamma^{2} \mathrm{e}^{-\gamma^{2}} \int_{0}^{\infty} \mathrm{d} y G(y)
$$

where

$$
\begin{aligned}
G(y)=2 & \sqrt{\frac{2}{3}} \frac{\mathrm{e}^{-3 y} \sinh \left\{3 \sqrt{y\left(y+2 \gamma^{2} / 3\right)}\right\}}{y \sqrt{y}} \\
& -\frac{8}{3} \gamma^{3} \frac{1}{y\left(y+2 \gamma^{2} / 3\right)}
\end{aligned}
$$

The integral of $G(y)$ has a finite value even if we take the limit of $\gamma \rightarrow 0$. Therefore eq 60 gives

$$
\begin{aligned}
I & =4 \sqrt{\pi} R \gamma^{2} \mathrm{e}^{-\gamma^{2}} \int_{0}^{\infty} \mathrm{d} y\left\{2 \sqrt{\frac{2}{3}} \frac{\mathrm{e}^{-3 y} \sinh (3 y)}{y \sqrt{y}}\right\} \\
& =16 \pi R \gamma^{2} \quad(\gamma \rightarrow 0)
\end{aligned}
$$

From eq 40, 55, and 62, we obtain the following simple result;

$$
k_{1}=\frac{1}{\sqrt{\pi}} \gamma
$$

Next, we evaluate the expansion coefficient $\xi_{1}$ in eq 48. From eq 49, we obtain

$$
\xi_{1}=-\sum_{n=0}^{\infty} \frac{A_{n}}{n-k_{1}} / \sum_{n=0}^{\infty} \frac{B_{n}}{\left(n-k_{1}\right)^{2}}
$$

Since $k_{1}$ is very small compared to unity we may approximate eq 64 as

$$
\xi_{1} \cong \frac{A_{0}}{k_{1}} / \frac{B_{0}}{k_{1}{ }^{2}}
$$

By use of eq 38, 40, and 63 , eq 65 gives

$$
\xi_{1}=\frac{\gamma}{4 \pi \sqrt{\pi} R^{2}}
$$

Substituting eq 63 and 66 into eq 48, we obtain the analytic expression for $\xi(\tau)$ with small $r$ as

$$
\xi(\tau)=\frac{\gamma}{4 \pi \sqrt{\pi} R^{2}} \exp \left(-\frac{\gamma}{\sqrt{\pi}} \tau\right) \quad(\gamma \rightarrow 0)
$$

and for $\chi(\tau)$ as

$$
\chi(\tau)=\exp \left(-\frac{\gamma}{\sqrt{\pi}} \tau\right)
$$

Hence, in the limit of $\gamma \rightarrow 0, \chi(\tau)$ exhibits a simple exponential decay for the whole timeregion. 


\section{COMPARISON WITH THE THEORY OF WILEMSKI AND FIXMAN}

Let us compare our results with that of Wilemski and Fixman. ${ }^{4}$ The system considered by Wilemski and Fixman is somewhat different from ours: they considered the case in which reactions occur with a certain second-order intrinsic rate constant $k$ if the reactive groups are within the distance $R$. The basic diffusion equation is then written as

$$
\frac{\partial}{\partial t} P(\boldsymbol{r}, t)=\mathscr{L} P-k S P
$$

where

$$
S(\boldsymbol{r})=\left(\frac{4 \pi R^{3}}{3}\right)^{-1} H(R-|\boldsymbol{r}|)=\left\{\begin{array}{cc}
\left(\frac{4 \pi R^{3}}{3}\right)^{-1} & (|\boldsymbol{r}| \leq R) \\
0 & (|\boldsymbol{r}|>R)
\end{array}\right.
$$

Eq 69 is more general than our eq 1 and 4 . In fact, for small $k$, eq 69 expresses the case where the reaction is not diffusion-controlled. On the other hand, in the limit of $k \rightarrow \infty$, eq 69 corresponds to our case of a purely diffusion-controlled reaction.

Their closure approximation yields the following form of $\hat{\chi}(p)$, the Laplace transform of the unreacted fraction of polymers.

$$
\hat{\chi}(p)=\frac{1}{p}-\frac{k v_{\mathrm{eq}}}{p^{2}\left\{1+\left(k / v_{\mathrm{eq}}\right) \hat{D}(p)\right\}}
$$

In eq $71, \hat{D}(p)$ is the Laplace transform of

$$
\begin{aligned}
D(\tau)= & \int \mathrm{d}^{3} u_{1} \int \mathrm{d}^{3} u_{2} S\left(\boldsymbol{u}_{1}\right) S\left(\boldsymbol{u}_{2}\right) G\left(\boldsymbol{u}_{1}, \boldsymbol{u}_{2}, \tau\right) P_{\mathrm{eq}}\left(\boldsymbol{u}_{2}\right) \\
= & \sum_{n=0}^{\infty} \int \mathrm{d}^{3} u_{1} \int \mathrm{d}^{3} u_{2} S\left(\boldsymbol{u}_{1}\right) S\left(\boldsymbol{u}_{2}\right) \\
& \times P_{\mathrm{eq}}\left(\boldsymbol{u}_{1}\right) \psi_{n}\left(\boldsymbol{u}_{1}\right) \psi_{n}\left(\boldsymbol{u}_{2}\right) P_{\mathrm{eq}}\left(\boldsymbol{u}_{2}\right) \exp (-n \tau)
\end{aligned}
$$

that is

with

$$
\hat{D}(p)=\sum_{n=0}^{\infty} \frac{D_{n}}{p+n}
$$

$$
\begin{aligned}
D_{n}= & \int \mathrm{d}^{3} u_{1} S\left(\boldsymbol{u}_{1}\right) \psi_{n}\left(\boldsymbol{u}_{1}\right) P_{\mathrm{eq}}\left(\boldsymbol{u}_{1}\right) \\
& \times \int \mathrm{d}^{3} u_{2} S\left(\boldsymbol{u}_{2}\right) \psi_{n}\left(\boldsymbol{u}_{2}\right) P_{\mathrm{eq}}\left(\boldsymbol{u}_{2}\right)
\end{aligned}
$$

and $v_{\text {eq }}$ is given by

$$
v_{\text {eq }}=\int \mathrm{d}^{3} u S(\boldsymbol{u}) P_{\text {eq }}(\boldsymbol{u})
$$

The reaction rate $k_{1}$ is the largest pole of eq 71 which can be evaluated by the root of the equation

$$
\hat{D}\left(-k_{1}\right)=\sum_{n=0}^{\infty} \frac{D_{n}}{n-k_{1}}=-\frac{v_{\text {eq }}}{k}
$$

since $p=0$ gives no singularity of $\hat{\chi}(p)^{4}$.

If we take the limit of $k \rightarrow \infty$ in eq 76 , we obtain

$$
\hat{D}\left(-k_{1}\right)=-\frac{D_{0}}{k_{1}}+\sum_{n=1}^{\infty} \frac{D_{n}}{n-k_{1}}=0
$$

Note that this equation has a close similarity to eq 50. The only difference is in the definitions of the coefficients $B_{n}$ and $D_{n}$. If we choose $S(\boldsymbol{u})=\delta(|\boldsymbol{u}|-R)$ in eq $74, D_{n}$ becomes equal to $B_{n}$ and eq 77 is reduced to eq 50 .

To see the quantitative difference, we solved eq 77 numerically for several values of $\gamma$. Substitution of eq 70 into eq 74 yields

$$
D_{n}=\left[3\left(\frac{3}{2 \pi L^{2}}\right)^{3 / 2} \int_{0}^{1} \mathrm{~d} x x^{2} \psi_{n}(R x) \mathrm{e}^{-\gamma^{2} x^{2}}\right]^{2}
$$

(Closure approximation)

Actually, Wilemski and Fixman employed a further simplification for the sake of computational convenience. They replaced one of the sink functions appearing in eq 74 by a delta function. In this case, we have

$$
D_{n}=3 P_{\mathrm{eq}}(0)\left(\frac{3}{2 \pi L^{2}}\right)^{3 / 2} \psi_{n}(0) \int_{0}^{1} \mathrm{~d} x x^{2} \psi_{n}(R x) \mathrm{e}^{-\gamma^{2} x^{2}}
$$

(Wilemski and Fixman)

To solve eq 77 with eq 78 or 79 , we employed the same numerical procedure as that of the solution of eq 50. The reaction rates for the above two cases, C.A. and W.F., are shown in Figure 5. In these cases, we can also obtain analytic expressions for $k_{1}$ in the limit of small $\gamma$. The detailed discussion will be given in a separate paper and the results are ${ }^{7}$

$$
\begin{array}{ll}
k_{1}=\frac{5}{6 \sqrt{\pi}} \gamma & \text { (C.A.) } \\
k_{1}=\frac{2}{3 \sqrt{\pi}} \gamma & \text { (W.F.) }
\end{array}
$$

These are also included in Figure 5. 


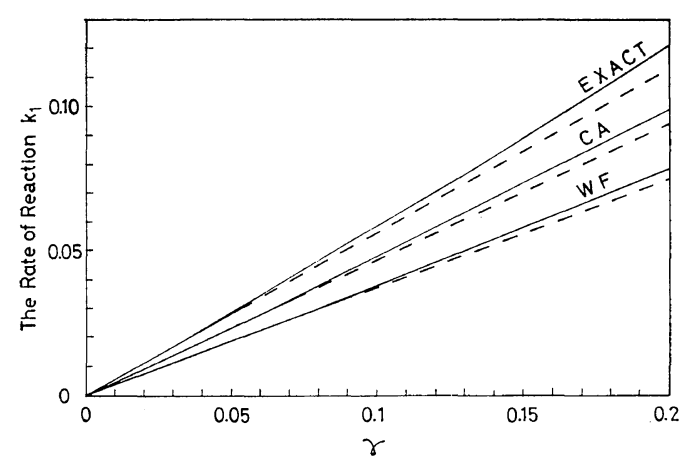

Figure 5. The rate of reaction: EXACT, solutions of eq 51; CA, solutions of eq 77 with eq 78; WF, solutions of eq 77 with eq 79. Dashed lines denote analytic expressions, eq 63,80 and 81 .

As is observed from Figure 5, the result of the closure approximation is relatively in good agreement with the exact one. The deviation is as much as $20 \%$, which will not be significant experimentally. The more important conclusion drawn from these calculations is that $k_{1}$ is approximately given by

$$
k_{1} \cong \gamma
$$

or

$$
k_{1} \cong \frac{R}{L} \frac{1}{\tau_{\mathrm{m}}}
$$

in the original time unit, where $\tau_{m}$ is the maximum relaxation time of the end-to-end vector of the harmonic spring model $\left(\tau_{\mathrm{m}}=L^{2} / 3 D\right)$. Eq 83 indicates that $k_{1}$ is smaller than $1 / \tau_{\mathrm{m}}$ by a factor $R / L$ and has a very simple physical meaning. Consider a free particle with a diffussion constant $D$, confined in a sphere of radius $L$. The particle is assumed to react with another particle located at the center if they are within the distance $R$. Then, the reaction rate for this system is estimated by ${ }^{6}$

$$
k_{1}=k_{2} n
$$

where $k_{2}$ is the second-order reaction rate constant predicted by the Smoluchowski theory and $n$ is the concentration. In this case, $k_{2}=4 \pi R D$ and $n=3 /\left(4 \pi L^{3}\right)$, thus we have eq 83 .

\section{DISCUSSION}

This paper has been devoted to an exact calculation of the reaction rates of the ring closure reaction of polymers based on the harmonic spring model. In this case, the reaction rates were proved to be almost proportional to $R / L$. In the limit of small $\gamma$, this proportionality has been shown analytically.

The discussion in the final part of the preceding section indicates that the spring potential affects only the restriction that both reactive segments must be within a distance $L$. The reaction rate of the harmonic spring model is well represented by one of free particles confined in a sphere of radius $L$. Hence, one may presume that the reaction rate for the Rouse chain is also given by eq 83 if $\tau_{\mathrm{m}}$ is understood as the longest relaxation time of the end-to-end vector. However, the validity of such a generalization remains in question. The results of the numerical calculation of Wilemski and Fixman show that, for the free draining Rouse chain, $k_{1}$ is almost independent of $R / L$. From the observation that the result of the closure approximation is in relatively good agreement with that of the exact one for the harmonic spring model, we suppose that this unexpected result comes from some essential differences between the harmonic spring model and the Rouse model. More detailed discussion will be given in a separate paper. ${ }^{7}$

Acknowledgment. The authors appreciate Dr. Gerald Wilemski of Dartmouth College for sending unpublished data.

\section{APPENDIX}

With the substitution of

$$
\begin{gathered}
P(\boldsymbol{r})=P_{\mathrm{eq}}(\boldsymbol{r}) \rho(\boldsymbol{r}) \\
P_{\mathrm{eq}}(\boldsymbol{r})=\left(\frac{3}{2 \pi L^{2}}\right)^{3 / 2} \exp \left(-\frac{3}{2 L^{2}}|\boldsymbol{r}|^{2}\right)
\end{gathered}
$$

eq 1 with eq 2 becomes

$$
\frac{\partial \rho}{\partial t}-D\left(\nabla^{2} \rho-\frac{3}{L^{2}} r \cdot \nabla \rho\right)=0
$$

To solve the eigenvalue problem associated with eq A3;

$$
\mathscr{D} \psi \equiv D\left(\nabla^{2}-\frac{3}{L^{2}} r \cdot \nabla\right) \psi=-\lambda \psi
$$

we transform eq A4 into the spherical coordinate. Since eq A4 is spherically symmetric, the eigenfunction is written as $\phi=R(r) Y_{l m}(\theta, \varphi)\left(Y_{l m}\right.$ being 
the spherical harmonics). Because of the sym- gives the desired results.

metrical reason of our system, we need only spherically symmetric eingenfunctions of eq A4 $(l=0)$. Then, eq A4 is reduced to

$$
\frac{\mathrm{d}^{2} R}{\mathrm{~d} r^{2}}+\left(\frac{2}{r}-\frac{3}{L^{2}} r\right) \frac{\mathrm{d} R}{\mathrm{~d} r}+\frac{\lambda}{D} R=0
$$

In terms of a new variable defined by

$$
\frac{3}{2 L^{2}} r^{2}=z
$$

eq A5 gives

$$
z \frac{\mathrm{d}^{2}}{\mathrm{~d} z^{2}} R(z)+\left(\frac{3}{2}-z\right) \frac{\mathrm{d} R}{\mathrm{~d} z}+\frac{L^{2}}{6 D} \lambda R=0
$$

Thus, $R(z)$ is identified with the Laguerre polynomials $L_{n}{ }^{1 / 2}(z)$;

$$
z \frac{\mathrm{d}^{2}}{\mathrm{~d} z^{2}} L_{n}{ }^{1 / 2}(z)+\left(\frac{3}{2}-z\right) \frac{\mathrm{d} L_{n}{ }^{1 / 2}}{\mathrm{~d} z}+n L_{n}{ }^{1 / 2}=0
$$

Then, the normalization of $\phi_{n}$

$$
\int \mathrm{d}^{3} r \psi_{n^{\prime}}(r) \psi_{n}(r) P_{\mathrm{eq}}(r)=\delta_{n^{\prime} n}
$$

$$
\begin{gathered}
\lambda_{n}=\frac{6 D}{L^{2}} n \quad(n=0,1,2, \ldots) \\
\psi_{n}(r)=\sqrt{\frac{\sqrt{\pi} n !}{2 \Gamma(3 / 2+n)}} L_{n}^{1 / 2}\left(\frac{3}{2 L^{2}} r^{2}\right)
\end{gathered}
$$

\section{REFERENCES}

1. J. C. Wang and N. Davidson, J. Mol. Biol., 19, 469 (1966).

2. N. Goodman and H. Morawetz, J. Polym. Sci., Part A-2, 9, 1657 (1971).

3. M. Sisido, Polymer J., 3, 84 (1972).

4. G. Wilemski and M. Fixman, J. Chem. Phys., 60, 886, 878 (1974).

5. R. M. Noyes, Progr. React. Kinet., 1, 129 (1961).

6. W. Strieder and R. Aris, "Variational Method Applied to Problems of Diffusion and Reaction," Springer-Verlag, Berlin-Heidelberg-New York, 1973, Chapter 3.

7. M. Doi, Chem. Phys., 9, 455 (1975). 